Meta

Journal des traducteurs

Translators' Journal

\title{
La littérature française traduite en Chine
}

\section{She Xiebin}

Volume 44, numéro 1, mars 1999

Théorie et pratique de la traduction en Chine

The Theory and Practice of Translation in China

URI : https://id.erudit.org/iderudit/004129ar

DOI : https://doi.org/10.7202/004129ar

Aller au sommaire du numéro

Éditeur(s)

Les Presses de l'Université de Montréal

ISSN

0026-0452 (imprimé)

1492-1421 (numérique)

Découvrir la revue

Citer cet article

Xiebin, S. (1999). La littérature française traduite en Chine. Meta, 44(1),

178-184. https://doi.org/10.7202/004129ar

\section{Résumé de l'article}

En Chine, la littérature française joue un rôle très important en traduction littéraire. Cet article est probablement le premier à présenter systématiquement aux lecteurs étrangers les traductions des oeuvres de la littérature française (fiction, poésie, théâtre, etc.) en Chine depuis la traduction, en 1899, par Linshu, de La Dame aux camélias d'Alexandre Dumas fils. On présente également de nombreux traducteurs chinois bien connus, dont la créativité ainsi que le travail acharné ont permis aux lecteurs chinois d'apprécier la brillante littérature française : les littératures du monde, les cultures et les civilisations peuvent ainsi s'entremêler harmonieusement.
Ce document est protégé par la loi sur le droit d'auteur. L'utilisation des services d'Érudit (y compris la reproduction) est assujettie à sa politique d'utilisation que vous pouvez consulter en ligne.

https://apropos.erudit.org/fr/usagers/politique-dutilisation/ 


\title{
La littérature française traduite en Chine
}

\author{
she xiebin \\ Université des Chemins de Fer de Changsha, \\ Changsha, Chine
}

\begin{abstract}
RÉSUMÉ
En Chine, la littérature française joue un rôle très important en traduction littéraire. Cet article est probablement le premier à présenter systématiquement aux lecteurs étrangers les traductions des œuvres de la littérature française (fiction, poésie, théâtre, etc.) en Chine depuis la traduction, en 1899, par Linshu, de La Dame aux camélias d'Alexandre Dumas fils. On présente également de nombreux traducteurs chinois bien connus, dont la créativité ainsi que le travail acharné ont permis aux lecteurs chinois d'apprécier la brillante littérature française: les littératures du monde, les cultures et les civilisations peuvent ainsi s'entremêler harmonieusement.
\end{abstract}

\section{ABSTRACT}

French literature plays a very important role in literary translation in China. Perhaps this paper has been the first to introduce systematically the translation and writing of French literature (fiction, poetry, drama, etc.) in China to the readers abroad since Linshu's translation of La dame aux camélias d'Alexandre Dumas fils in 1899. And an introduction is also given to a lot of well-known translators in China, by whose creative and painstaking working the Chinese readers have the chance to appreciate the brilliant French literature, and world literatures, cultures and civilizations get well mingled.

La littérature française occupe une place importante dans la littérature étrangère traduite en chinois. Elle a exercé, et exerce encore, par sa richesse, une grande influence sur le développement de la littérature chinoise. Parmi les œuvres littéraires françaises traduites en chinois, on peut trouver des romans, des pièces de théâtre, des poèmes et d'autres genres de composition littéraire de toutes les époques (du M oyen Âge au $x x^{e}$ siècle) et de toutes les écoles (I'humanisme, le classicisme, le structuralisme, l'absurde, etc.). Les 200 écrivains français les plus célèbres enregistrés dans la Grande Encyclopédie de Chine ont été présentés plus ou moins, ou traduits totalement ou partiellement en chinois.

Traduire est une chose difficile. Traduire du français au chinois est plus difficile encore, étant donné les grandes différences entre les deux langues et les deux cultures. C'est grâce à un travail assidu, créatif et inlassable qui demande beaucoup de courage, de volonté, de sensibilité et d'intelligence de la part des traducteurs que le vaste public chinois connaît de grands écrivains français ainsi que leurs chefs-d'œuvre.

\section{LES ROMANS FRANÇAIS TRADUITS EN CHINOIS}

Les plus importants romanciers français traduits en chinois sont: Guy de Maupassant, Anatole France, Alphonse Daudet, Victor Hugo, Voltaire, Gustave Flaubert, Prosper M érimée, Alexandre Dumas père, Alexandre Dumas fils, Honoré de Balzac, 
Émile Zola, Stendhal, Romain Rolland, François Rabelais, Denis Diderot, Montesquieu, Alain René Lesage, George Sand, Louis Aragon, Georges Duhamel, André Stil, Delly, Henri Barbusse, Paul Vaillant-Couturier, Jean-Jacques Rousseau, Alfred de Musset, Jules Vallès, Jules Verne, François M auriac, Albert Camus, Antoine de SaintExupéry, M arcel Aymé, Choderlos de Laclos, abbé Prévost, etc. Ces dernières années, M M. Liu M ingjiu, Jin Zhiping et Luo Xinzhang, chercheurs et traducteurs de littérature française, ont dirigé la rédaction d'une Collection des œuvres littéraires françaises du $x x^{e}$ siècle. Les principaux écrivains français contemporains traduits sont: André Maurois, Marguerite Duras, Marguerite Yourcenar, Jean-Paul Sartre, Alain RobbeGrillet, André M alraux, Hervé Bazin, Henry de Montherlant, André Gide, Françoise Sagan, Jean-Louis Curtis, Colette, Georges Bernanos, Raymond Radiguet, Roger Vailland, etc.

Je regrette de ne pas pouvoir citer ici tous les romanciers français déjà connus en Chine ou traduits en chinois. Je peux dire cependant qu'en Chine on suit toujours de près ce qui se passe dans les milieux littéraires français. II existe, dans les instituts d'études de littérature étrangère ou les universités, un bon contingent de chercheurs et de professeurs qui étudient sous différentes formes les écrivains français.

\section{La première traduction de roman}

La Dame aux camélias d'Alexandre Dumas fils, traduit en langue classique chinoise en 1899 par Lin Shu (Lin Qinnan 1852-1924), était la première traduction d'une œuvre de littérature étrangère en chinois. La parution de ce roman a connu un grand succès et une répercussion générale. C'est probablement grâce à son influence directe ou indirecte que les romanciers chinois ont écrit une série de romans d'amour tout différents de ceux des classiques. Pourtant, le texte traduit ne correspondait pas fidèlement à l'original, car Lin Shu ne connaissait pas le français ni aucune autre langue étrangère, il a traduit d'après la narration d'un interprète du nom de Wang Shouchang qui avait fait ses études à Paris. Naturellement, ce roman a été retraduit plusieurs fois par la suite.

\section{Le roman le plus difficile à traduire en chinois}

À la recherche du temps perdu, de Marcel Proust, est un des romans français les plus connus du monde. L'originalité de sa forme artistique, la profondeur de sa signification, son étrangeté d'expression et son souvenir dépassant l'espace et le temps en rendent la traduction très difficile (voire parfois impossible), tant pour la compré hension que pour l'expression en chinois. C'est grâce à un effort collectif que cet ouvrage a été traduit en chinois, en 1994, par 15 professeurs des universités pour le compte des Éditions de Yilin. On doit reconnaître que cette traduction a eu beaucoup de succès, mais on y trouve encore des insuffisances surtout dans l'expression du style du texte original, à cause de la diversité des personnalités des 15 traducteurs.

\section{Le roman le plus retraduit en chinois}

La retraduction des œuvres littéraires étrangères classiques est devenue un phénomène remarquable en Chine. Prenons, par exemple, le chef-d'œuvre de Stendhal, Le 
Rouge et le Noir, qui a été traduit pour la première fois en 1947 par Zhao Ruihong. Dès lors, ont paru successivement près d'une vingtaine de versions retraduites par différents traducteurs, dont voici les plus connues:
version retraduite par Luo Yujun en 1954; version retraduite par $\mathrm{H}$ ao Yun en 1986; version retraduite par Wen Jiasi en 1988; version retraduite par Guo Hongan en 1993; version retraduite par Xu Yuanchong en 1993; version retraduite par Luo Xinzhang en 1994.

La retraduction de ce célèbre roman français a suscité, au cours des dernières années, la plus «grande tempête d'encre» (discussion et critique des traductions) qu'a jamais connue la Chine et qui a pour but ultime de fournir une version chinoise pouvant soutenir la comparaison avec l'original.

\section{Quelques romanciers français les plus traduits en chinois}

Voici quelques romanciers français parmi les plus connus des lecteurs chinois, grâce à la traduction de leurs ouvrages.

Victor Hugo: ses principaux romans traduits en chinois sont NotreDame de Paris, Les Misérables, L'H omme qui rit, Quatre vingt-treize, etc.

H onoré de Balzac: ses principaux romans traduits en chinois sont Eugénie Grandet, Le Père Goriot, Les Paysans, La Cousine Bette, Le Curé de Tours, Histoire de la grandeur et de la décadence de César Birotteau, Le Cousin Pons, etc. Ses quatre nouvelles (Adieu, JésusChrist en Flandre, L'Auberge Rouge et Réquisitionaire) ont été traduites en 1915 par Lin Shu et Chen Jialin. La première traduction du roman Eugénie Grandet a vu le jour en 1936, traduit par Mu Mutian.

Alexandre Dumas père: ses principaux romans traduits en chinois sont Le Comte de M onteCristo, Les Trois M ousquetaires, etc.

George Sand: ses principaux romans traduits en chinois sont La M are au diable, La Petite Fadette, François le champi, Le M eunier d'Angibault, Contes d'une grand'mère, etc.

Jules Verne: ses principaux romans d'aventures ont été traduits en chinois, tels que Cinq semaines en ballon, Les Aventures du capitaine Hatteras, Vingt mille lieues sous les mers, Voyage au centre de la Terre, De la Terre à la Lune, Les Enfants du capitaine Grant, L'île mystérieuse, Le Tour du monde en 80 jours, etc.

Émile Zola: ses principaux romans traduits en chinois sont La Fortune des Rougon, L'Assommoir, Nana, Au bonheur des dames, Germinal, L'Argent, La Débâde, etc.

Guy de M aupassant: ses principaux romans traduits en chinois sont Bel-ami, Une vie, M ontOriol, N otre couur, Fort comme la mort, Boule de suif, etc. En 1991, les Éditions littéraires et artistiques du Hunan ont édité N ouvelles complètes de M aupassant traduites par Li Qinya.

Romain Rolland: ses principaux romans traduits en chinois sont Jean-Christophe, Colas Breugnon, L'Âme enchantée, etc.

\section{Les traducteurs les plus connus}

En près d'un siècle a surgi un grand nombre de traducteurs des romans français, dont les plus remarquables sont:

Fu Lei (1908-1966), connu pour la traduction de 14 romans de Balzac et du chef d'œuvre de Romain Rolland, Jean-Christophe. Il était membre de l'Association d'études de Balzac de France. 
Li Qinya (1884-1969), connu pour la traduction des ouvrages de M aupassant, de Flaubert, de Voltaire, d'Anatole France, de Daudet et d'Alexandre Dumas père.

Li Dan (1901-1976), connu pour la traduction des M isérables de Victor Hugo.

Luo Yuzhun (1870-?), connue pour la traduction des œuvres littérai res de George Sand et du Rouge et le Noir de Stendhal.

On pourrait encore citer beaucoup d'autres traducteurs comme Li Jianwu, Zhao Shaohou, Luo Dagang, Gao Mingkai, Mu Mutian, Chen Zhanyuan, Chen Jingrong, Zheng Yonghui, Lu Ying, Qi Fang, Hao Yun, Bi Xiushao.

\section{LA POÉSIE FRANÇAISE TRADUITE EN CHINOIS}

La traduction de la poésie française en chinois a une histoire de plus de 120 ans. La Marseillaise traduite en 1871 par Wang Tao a été peut-être la première traduction disponible. Dès lors, Ma Junwu, Su M anshu, Gao Junping, Liu Bannong ont traduit successivement en chinois classique un bon nombre de poèmes français célèbres. C'est après le M ouvement du 4 M ai 1919, et surtout après la fondation de la République populaire de Chine, que la traduction des poèmes français a connu un grand développement.

\section{Les principaux poètes français traduits en chinois}

La France est un pays dont la poésie a influencé et influence encore les poètes du monde. En Chine, on a présenté et traduit un grand nombre des poètes français les plus connus, tels que M aurice Scève, Pierre de Ronsard, François de $M$ al herbe, Honorat de Racan, Fr. De Maynard, Nicolas Boileau-Despréaux, Jean Racine, Jean de La Fontaine, Voltaire, André Chénier, Alfred de Vigny, Leconte de Lisle, Sully Prudhomme, François Coppée, José-M aria de Heredia, $M{ }^{m e}$ Ackermann, Albert Samain, M aurice Maeterlinck, Alphonse de Lamartine, Arthur Rimbaud, Paul Verlaine, Paul Claudel, Victor Hugo, Francis Jammes, Guillaume Apollinaire, J. Gautier, Paul Valéry, Charles Péguy, Max Jacob, Léon-Paul Fargue, Valery Larbaud, C. Pozzi, André Salmon, Jules Supervielle, Saint-John Perse, Pierre Reverdy, Henri M ichaux, Robert Desnos, Francis Ponge, René Char, André Frénaud, Patrice de la Tour du Pin, Yves Bonnefoy, Jacques Dupin, J. M alaquais, etc.

Les principaux traducteurs de ces poètes français sont: Dai Wangshu, Liang Zongdai, Shen Baoji, Bian Zhilin, Shi Zhecun, Mu M utian, Chen Jingrong, Wen Jiasi, Luo Dagang, Zhang Yinlun, Wang Liaoyi, Fan Xiheng, Qian Chunqi, Cheng Baoyi, Luo Luo, Li Hengji, Xu Zhimian, Jin Zhiping, Guo Hongan, Chen Chengrong, Hong Zhongpu, Xu Deyan, Fang Jing, Ge Lei, Zhang Guangyao, Shen Zhiming, etc.

Outre les poèmes français traduits et publiés par les revues ou les journaux chinois, les principaux recueils de poèmes tirés à part sont:

recueil de poèmes de Victor Hugo ;

recueil de poèmes de Pierre Jean de Béranger;

recueil de poèmes de Pottier;

recueil de poèmes d'Alfred de Musset;

recueil de poèmes de Paul Éluard ;

recueil de poèmes de Louis Aragon ;

recueil de poèmes de la Commune de Paris; 
recueil de poèmes de Charles Baudelaire (Les Fleurs du mal) ;

recueil de poèmes de la Pléiade;

recueil de poèmes contemporains français;

recueil de poèmes modernes français;

recueil de poèmes romantiques français;

recueil de poèmes lyriques français;

recueil de poèmes d'amour français, etc.

\section{Shen Baoji, éminent traducteur de la poésie française}

On dit que seul le poète peut traduire les poèmes. Je ne partage pas un point de vue aussi absolu, mais il est vrai qu'un poète a des qualités innées pour traduire un poème. Dai Wangshu, Liang Zongdai et Shen Baoji en sont de bons exemples. Ils sont tout à la fois poètes et traducteurs et ils nous ont donné un grand nombre de belles traductions de poèmes français.

C'est avec grand plaisir que je présente ici $M$. Shen Baoji, qui est à la fois mon professeur et mon collègue et qui, âgé de 89 ans, travaille encore pour la diffusion de la littérature française.

Né le 5 avril 1908 dans la province du Zhejiang, Shen Baoji a commencé ses études de langue et de littérature françaises à l'âge de 14 ans, d'abord dans une école sino-française de Shanghai puis à I'U niversité de l'Aurore dirigée par les missionnaires catholiques. En 1925, il est entré à I'Institut franco-chinois de Beijing (Pékin). Grâce à ses trois ans d'études assidues, il s'est classé parmi les premiers et a obtenu ainsi une bourse pour aller étudier à l'Université de Lyon pendant 6 ans, de 1928 à 1934 ; c'est là qu'il a obtenu son doctorat ès lettres avec une thèse intitulée Études de Si Siang-ki (une des plus célèbres pièces du théâtre classique chinois) rédigée en français et publiée en France.

Après avoir fini ses études en France, Shen Baoji a regagné son pays et a enseigné le français et la littérature française à I'Institut franco-chinois de Beijing. II était alors un des rédacteurs d'Études françaises, revue dirigée par la Commission de l'édition culturelle sino-française, dont André d'Hormon (qui a enseigné le français pendant 49 ans en Chine et qui a révisé la traduction du Rêve dans le pavillon rouge après son retour en France) était le rédacteur en chef. Dans cette revue, Shen Baoji a publié une série de traductions, d'essais et de commentaires sur la littérature française (surtout la poésie) dont la plupart des auteurs étaient inconnus à cette époque des lecteurs chinois.

Après la fin de la guerre sino-japonaise, invité par Xu Beihong (grand peintre chinois), Shen Baoji a travaillécomme professeur de français et d'art à l'École spéciale de l'Art de Beijing. Puis il a travaillé à l'Université de Beijing (Pékin), à l'Institut de géologie de Wuhai, à I'Institut des Chemins de fer de Changsha. Au cours d'une trentaine d'années d'enseignement, il a formé un grand nombre d'étudiants dont beaucoup sont devenus professeurs, interprètes ou traducteurs, qui travaillent maintenant à l'intérieur comme à l'extérieur de la Chine. Le traducteur du Rêve dans le pavillon rouge, Li Zhihua, était un de ses élèves.

Passionné de langue et de littérature françaises, Shen Baoji a traduit, tout en enseignant, un grand nombre de poèmes français et a rédigé une série d'essais et de commentaires sur la poésie française, publiés dans divers journaux et revues chinois. D'après des statistiques incomplètes, Shen Baoji a fait connaître, pendant près de 
60 ans, une centaine de poètes et d'écrivains et a traduit plus de 700 poèmes français. C'est grâce à ses belles traductions et à ses études approfondies que le peuple chinois peut avoir accès, souvent pour la première fois, aux beaux poèmes de Victor Hugo, de Béranger, d'Apollinaire, des poètes de la Commune de Paris, etc.

Influencé par le symbolisme et le surréalisme français dès sa jeunesse, Shen Baoji a écrit environ 800 poèmes en chinois, dont une bonne partie a été publiée. II entretenait des relations amicales avec le célèbre poète $D$ ai Wangshu. Et il était un des deux directeurs d'études pour les jeunes poètes chinois pendant les années 30, I'autre directeur étant le grand savant et poète Zhu Guangqian.

Voici un de ses poèmes, écrit lors de son $80^{e}$ anniversaire, que j'ai essayé de traduire en francais.

Vieux, je suis bien vieux

Pourtant l'affaiblissement physique

Est peu de chose à mes yeux

Vieux, je suis bien vieux

Pourtant une source vive

Coule dans mon cœur

La plus grande tristesse

C'est d'avoir une pensée qui se meurt

Vieux, je suis bien vieux

Pourtant ceux qui me connaissent

Disent que j'ai une ardeur juvénile

Et que mes vers sont merveilleux

- ô non ô non

Seules les amitiés authentiques

Sont un poème vraiment beau

Vieux, je suis bien vieux

Pourtant dans ma vieillesse

Surgit une troisième enfance

Je suis donc encore un enfant

Vieux, je suis bien vieux

Pourtant au-delà des mers

Comme dans mon pays natal

Jamais je ne me sens solitaire

La plus grande joie

C'est d'avoir des amitiés profondes

Vieux, je suis bien vieux

Pourtant après le grand désastre

Apparaît une époque prospère

Où les savoirs sont respectés

De mieux en mieux

Je comprends qu'il faut me souvenir

De mon passé et y longuement réfléchir

Vieux, je suis bien vieux

Pourtant prenons pour exemple

Le vieux coursier qui refuse de se reposer

Et le ver à soie filant son cocon

Jusqu'au dernier moment 


\section{LES PIÈCES DE THÉÂTRE FRANÇAIS TRADUITES EN CHINOIS}

La traduction des pièces de théâtre français en chinois date de 1910. Pourtant, avant la traduction écrite, on avait déjà mis en scène une série de pièces de théâtre français, dans des écoles dirigées par des missionnaires catholiques étrangers, d'après la pré sentation de l'intrigue. La Dame aux camélias d'Alexandre Dumas fils a été peut-être la première pièce de théâtre étrangère mise en scène en Chine, et a entraîné une grande révolution du théâtre traditionnel chinois.

Les principaux écrivains de pièces de théâtre français traduites en chinois sont: Pierre Corneille, M olière, Jean Racine, Beaumarchais, Honoré de Balzac, Alain René Lesage, Prosper M érimée, Alfred de Musset, Alexandre Dumas fils, Romain Rolland, Jean-Paul Sartre, etc.

Les principaux traducteurs des pièces de théâtre français sont: Liu Bannong, Chen Mian, Wu wenjiang, Qi Fang, Li Jianwu, Zhao Shaohou, Deng Lin, Chen Ji, Wan Xin, Wang Liaoyi, Wu Dayuan, Chen Xuezhao, Li Cangren, Chen Souzhou, Quo Linge, Li Liewen, Sun Fuxi, Shen Qiyu, Wang Zhensun, etc. Parmi eux, le plus célèbre est Li Jianwu, qui a traduit 27 pièces de Molière.

La littérature française traduite en chinois est un grand thème de recherche. $\mathrm{Ce}$ quej'ai présenté ici est loin d'être complet. Et encore, je ne parle pas de la traduction des autres genres de composition littéraire qui constituent aussi une partie des grands travaux de nos traducteurs chinois. Pourtant, comme dit un proverbe chinois: «En regardant un léopard à travers un tuyau de bambou, on peut quand même en voir une tache. » On peut avoir aussi, à travers mon article, une idée d'ensemble sur la grande contribution que les traducteurs chinois ont apportée à la présentation de la littérature française en Chine.

\section{RÉFÉRENCES}

M a, Zuyi (1984) : Précis de l'histoire de la traduction chinoise, Beijing.

Chen, Fukang (1992) : D ocument historique de la traduction chinoise, Shanghai.

Chen, Zongbao (1984) : Cours de traduction du français en chinois, Shanghai.

Catalogue des ouvrages littéraires classiques étrangers traduits et publiés en Chine de 1949 à 1979

(1980) : Bibliothèque d'édition nationale du Bureau d'administration des publications, Beijing.

Lin, Hui (1988) : Dictionnaire des traducteurs chinois, Beijing.

She, Xiebin (1996) : «Les romans français traduits en chinois», Chinese Translators Journal, n 1.

- - (1996) : «La Poésie française traduite en chinois», Foreign Language Teaching and Researh, $\mathrm{n}^{\circ} 2$.

- - (1996) : «Shen Baoji, éminent traducteur chinois de la littérature française», Recueil des essais d'études des langues étrangères, Beijing.

Xu , Jun (1996) : Écriture, littérature et culture: études des traductions chinoises du Rouge et le Noir, Nanjing. 